\title{
A matter of life and death for caspase 8
}

Cells can control their own death as part of normal development or

These studies... describe a previously unknown mechanism for catalytically inactive caspase 8 in triggering the pyroptosis cell death pathway in the absence of apoptosis and necroptosis

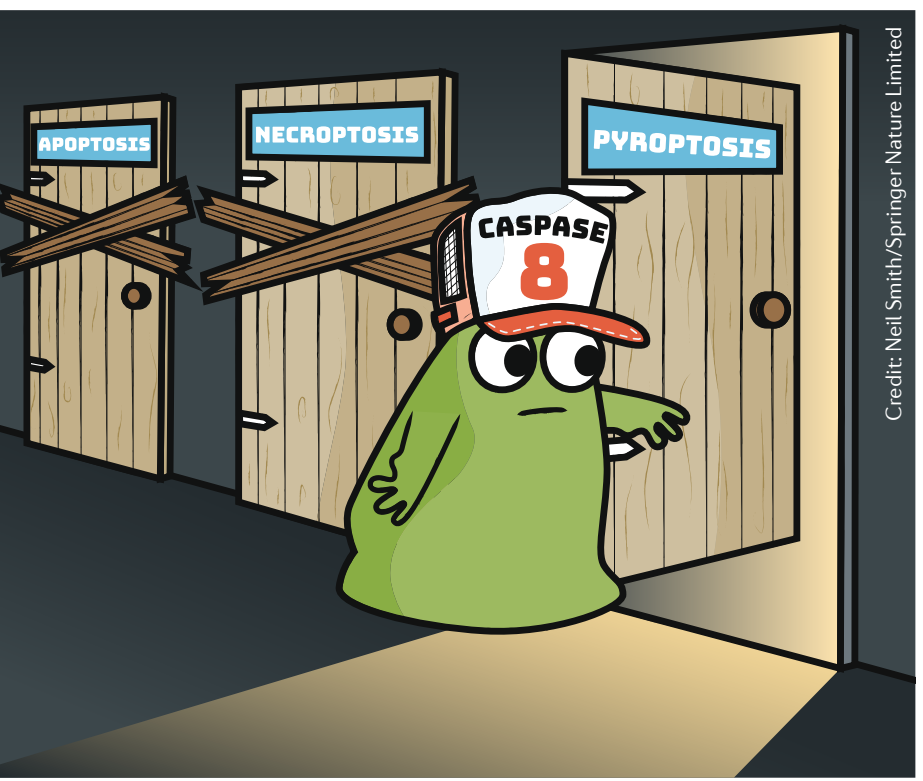

day (E)11.5 and used different models to look for the cause of embryonic lethality. Fritsch et al. expressed caspase 8 with a point mutation in its substrate binding pocket $\left(\right.$ Casp $\left.8^{\mathrm{C} 362 \mathrm{~S} / \mathrm{I}}\right)$ in epidermal keratinocytes and in intestinal epithelial cells (using Krt14 ${ }^{c r e}$ and Villin $^{\text {cre }}$ drivers, respectively). These mice were viable but experienced necroptosis in these tissues. Suppression of necroptosis through deletion of $M l k l$ rescued the skin phenotype but worsened the inflammatory destruction of the intestine, leading to premature death of $\operatorname{Casp} 8^{\mathrm{C} 362 \mathrm{~S} / \mathrm{l} l}$ Villin $^{\text {cre }}$ mice at 4 weeks of age. Moreover, Casp $8^{\mathrm{C} 3625 / 362 S}$ mice deficient in the necroptosis mediator RIPK3, while not showing embryonic lethality, were dramatically stunted, suggesting a necroptosis-independent role for inactive caspase 8 . The authors examined soluble proteins in the ileum of $\operatorname{Casp}^{\text {C362S/fl }}$ Villin $^{\text {cre }} \mathrm{Mlk}^{-/-}$ mice and found high levels of IL- $1 \beta$ and the active cleaved version of the executioner caspase caspase 1. Cleavage of caspase 1 and IL- $1 \beta$ are signs of pyroptotic cell death, suggesting pyroptosis of ileal epithelial cells in the absence of necroptosis.

In a concurrent study, Newton et al. showed that knockout of $M l k l$ in mice with a catalytically inactive caspase $8\left(\right.$ Casp $\left.^{\mathrm{C} 362 \mathrm{~A} / \mathrm{C} 362 \mathrm{~A}}\right)$ reversed embryonic death, but caused death during the perinatal period. Early embryos of Casp $8^{362 A / 362 A}$ $M l k l^{-/-}$mice expressed higher levels of inflammatory cytokines in the gut than $\mathrm{Casp}^{-/-} \mathrm{Mlk^{-/- }}$ embryos. Using multiple knockout models to investigate the role of caspase 1 , caspase 11 and the necroptosis protein RIPK3 in the Casp8 $8^{\mathrm{C} 362 \mathrm{~A} / \mathrm{C} 362 \mathrm{~A}}$ $M l k l^{-/}$phenotype, the authors found caspase 1 to be a major driver for perinatal lethality in Casp8 $8^{\mathrm{C} 362 \mathrm{~A} / \mathrm{C} 362 \mathrm{~A}} \mathrm{Mlkl}^{-/-}$mice, with caspase 11 contributing to death around weaning. These data suggest that pyroptosis mediates perinatal death. Although caspase 1 was driving lethality, knockout of the pyroptosis pore-forming protein and caspase 1 substrate gasdermin D did not rescue $\operatorname{Casp} 8^{\mathrm{C} 362 \mathrm{~A} / \mathrm{C} 362 \mathrm{~A}} \mathrm{Mlkl^{-/ }}$ mice from dying shortly after birth, suggesting that cleavage of other caspase 1 substrates, such as caspase 3 or caspase 7 , may drive perinatal lethality.

Both studies found that the expression of inactive caspase 8 or pan-caspase inhibition induced the formation of apoptosis-associated speck-like protein containing a CARD (ASC) specks and subsequent caspase 1 activation. Each group also showed that deletion of ASC or caspase 1 rescued perinatal lethality in caspase 8 activity-deficient $M l k l^{-/-}$mice, suggesting that an ASC inflammasome-driven mechanism causes lethality in the absence of active caspase 8 .

These studies therefore describe a previously unknown mechanism for catalytically inactive caspase 8 in triggering the pyroptosis cell death pathway in the absence of apoptosis and necroptosis. This mechanism could act as a defence against viruses that have evolved strategies to inhibit caspase 8 catalytic activity and parts of the necroptosis machinery.

\section{Joseph Willson}

ORIGINAL ARTICLES Newton, K. et al. Activity of caspase-8 determines plasticity between cell death pathways. Nature 575, 679-682 (2019) | Fritsch, M. et al. Caspase- 8 is the molecular switch for apoptosis, necroptosis and pyroptosis. Nature 575, 683-687 (2019) 